\title{
Development and Optimization of w/o/w Multiple Emulsion of Lisinopril Dihydrate Using Plackett Burman and Box-Behnken Designs
}

\section{Sawant KK*, Mundada VP and Patel VJ}

Drug Delivery Laboratory, Centre of Relevance and Excellence in NDDS, Pharmacy Department, The Maharaja Sayajirao University of Baroda, Donor's Plaza, Fatehgunj, Vadodara-390002, India

\begin{abstract}
The aim of the present study was to develop water-in-oil-in-water (w/o/w) type Multiple Emulsion of Lisinopril dihydrate for enhancing its oral bioavailability via enhanced permeation. For primary emulsification, corn oil was used as the oil phase, Span 83 as the lipophilic surfactant and Xanthan gum was used as the viscosity enhancer. Primary emulsion was re-emulsified with aqueous phase containing Tween 20 as hydrophilic surfactant. Preliminary screening was performed using a 12-run, 8-factor, 2-level Plackett-Burman design followed by Box Behnken Design for optimization. MEs were characterized and evaluated for macroscopic and microscopic properties, globule size, entrapment efficiency, rheological properties, in vitro and ex vivo drug release and stability studies. In vitro drug diffusion study was done through dialysis bag and ex vivo permeability studies were performed in Franz diffusion cell using rat intestine. The optimized w/o/w ME showed globule size and entrapment efficiency of $15.65+1.967 \mu \mathrm{m}$ and $87.35+$ $3.79 \%$ respectively. Drug flux was found to be $119.3 \mu \mathrm{g} / \mathrm{cm} 2 / \mathrm{h}$ for drug loaded w/o/w ME and $105.1 \mu \mathrm{g} / \mathrm{cm} 2 / \mathrm{h}$ for plain drug solution. The overall results of the studies showed the potential of the w/o/w ME as promising drug delivery system for Lisinopril dihydrate.
\end{abstract}

Keywords: Lisinopril dihydrate; Water-in-Oil-in-Water Multiple emulsion; Optimization; Plackett Burman design; Box-Behnken design; Permeability; Flux

\section{Introduction}

Multiple emulsion (ME) systems are novel developments in the field of emulsion technology. MEs are complex systems, termed as emulsions of emulsions, in which globules of the dispersed phase encapsulates smaller droplets, which may normally consist of a liquid miscible with and in some cases identical with, the continuous phase. This is made possible by double emulsification; hence the systems are also called as 'double emulsion'. Each dispersed globule in the double emulsion forms a vesicular structure with single or multiple compartments separated from the aqueous phase by a layer of oil phase compartment [1]. Inherent instability is the main problem with respect to MEs. To stabilize the system, various emulsifiers can be used. W/O emulsion can be stabilized by low HLB or oil soluble surfactants whereas O/W emulsions can be stabilized by high HLB or water soluble surfactants. Intermediate HLB can provide systems with optimal stability. MEs may be prepared by using such pairs of surfactants which will impart some degree of stability [2]

Double emulsions are helpful to maintain drug concentrations within therapeutic range in the lymph vessels. The transport of drugs to the mesenteric lymph nodes rather than into the portal system directly is due to encapsulation of the drug by the oil phase, which provides more of lipophilic environment and therefore are absorbed through lymphatic vessels. In addition, w/o/w emulsions might act as carriers for the delivery of polypeptide/protein drugs, which require both protection from the gastric fluids and delivery via the lymph nodes [3]. ME's potential biopharmaceutical applications also incorporates intestinal [4] and prolonged [5] drug delivery. Shichiri et al. developed w/o/w MEs for oral administration of insulin by protecting it from proteolytic destruction and facilitating intestinal absorption [6]. MEs are expected to allow delivery of anticancer agents by sparing normal tissues and selectively attacking tumour tissues [7]. Kim et al. studied Cytarabine-loaded w/o/w multiple emulsions using nonionic surfactants of the Tween and Span types. The release study showed that the multiple emulsion containing cytarabine in the internal aqueous phase was stable, exhibiting a prolonged release pattern [8]

Onuki et al. had successfully prepared and optimized the formulation with the highest stability and the most desirable pharmacological effect of the water-in-oil-in-water multiple emulsion for intestinal insulin delivery based on statistical methods such as the orthogonal experimental design and the response surface evaluation [9].

Our research group has previously developed w/o/w type multiple emulsions entrapping acyclovir for improving its oral bioavailability. Particle size of $33.098 \pm 2.985 \mu \mathrm{m}$ and entrapment efficiency of 85.25 $\pm 4.865 \%$ were obtained. Drug release from the prepared formulations showed initial rapid release followed by a much slower release. In vivo studies in rats indicated prolonged release and better oral bioavailability as compared to drug solution [10].

Lisinopril dihydrate (LD) a synthetic peptide derivative, is an oral long-acting angiotensin converting enzyme (ACE) inhibitor [11] widely used for the treatment of hypertension caused by increased plasma level of angiotensin II. LD is a Biopharmaceutical Classification System (BCS) class III drug [12], exhibiting variable absorption (6 to $60 \%$ ) and poor bioavailability (30\%) [13]. Hence, the present investigation was aimed at preparation and optimization of w/o/w Multiple Emulsion of LD with the objective of improving its oral bioavailability (via protection in gastric environment and lymphatic delivery).

*Corresponding author: Krutika K Sawant, Drug Delivery Laboratory, Centre of Relevance and Excellence in NDDS, Pharmacy Department, The Maharaja Sayajirao University of Baroda, Donor's Plaza, Fatehgunj, Vadodara-390002, India, Tel: +912652434187; Fax: +912652423898; E-mail: dr_krutikasawant@rediffmail.com

Received January 09, 2017; Accepted February 06, 2017; Published February 15, 2017

Citation: Sawant KK, Mundada VP, Patel VJ (2017) Development and Optimization of w/o/w Multiple Emulsion of Lisinopril Dihydrate Using Plackett Burman and Box-Behnken Designs. J Nanomed Nanotechnol 8: 422. doi: 10.4172/2157 7439.1000422

Copyright: (c) 2017 Sawant KK, et al. This is an open-access article distributed under the terms of the Creative Commons Attribution License, which permits unrestricted use, distribution, and reproduction in any medium, provided the original author and source are credited. 
The purpose of optimization of any pharmaceutical process is to determine and evaluate independent variables that affect formulation response. Using independent variables, best response values with maximum desired characteristics can be developed with minimum batches to reduce the cost of final product [14]. The number of trials can be reduced by using combination of design tools. In this work, we employed well established statistical tools viz., Plackett-Burman for initial screening and Box Behnken Design, a sub type of response surface methodology for optimization of processing conditions and to identify multi factor interactions [15]. Plackett-Burman Design (PBD) is more practical and frequently used approach whenever a large number of variables are involved [16]. Hence to test a large number of variables with fewer experimental runs, Plackett-Burman Design (PBD) was selected for preliminary screening. Since there is limitation in $\mathrm{PBD}$ that it does not provide information regarding the interaction effects between different variables, it is of low utility in the main optimization stage. Therefore, Box-Behnken Design (BBD) of high resolution was used to optimize final stages of ME. Response Surface Methodology (RSM) [17] was selected to identify multi-factor interactions and optimise the processing conditions in the preparation of multiple emulsion. The Plackett-Burman screening design was constructed using Minitab version 17 (Minitab Inc., State College, PA) and RSM-Box Behnken Design using Design Expert 7.0.0 software.

\section{Materials and Methods}

\section{Materials}

Lisinopril dihydrate was received as a gift sample from Torrent Research Centre, Ahmedabad, India. Span 80, Tween 20 and Xanthan gum were obtained from S.D. Fine Chemicals Ltd., Mumbai, India. Span 83 and Corn oil were obtained from National Chemicals, Vadodara, India. Other chemical reagents and solvents used were of Analytical Reagent (A.R) or HPLC grade.

\section{Screening of oil}

Oil was selected on the basis of solubility of LD in various oils. Different oils like light liquid paraffin, Soyabean oil, Sunflower oil, Linseed oil and Corn oil were screened. $10 \mathrm{~mL}$ of the selected oil was added to each cap vial containing an excess of LD (100 mg). After sealing, mixtures were shaken for $15 \mathrm{~min}$ and were kept at $25^{\circ} \mathrm{C}$ for 48 h. After reaching equilibrium, the vials were centrifuged at $3000 \mathrm{rpm}$ for $5 \mathrm{~min}$. The supernatant was separated carefully and analyzed for LD content after appropriate dilutions with dimethylformamide (DMF) using UV-spectrophotometer (UV-1800 Shimadzu, Japan) at $595 \mathrm{~nm}$ [18].

\section{Preparation of ME}

Two-step emulsification technique was used to prepare ME.

- Primary emulsification: $4 \mathrm{~mL}$ of aqueous phase containing $20 \mathrm{mg}$ of $\mathrm{LD}$ and $0.2 \% \mathrm{w} / \mathrm{v}$ of Xanthan gum was added to 6
$\mathrm{mL}$ of corn oil containing 10\% w/v of Span 80 or Span 83 and emulsified at $6500 \mathrm{rpm}$ for $3 \mathrm{~min}$ using Ultra-Turrax T-25 (IKA, India) homogenizer.

- Secondary emulsification: $10 \mathrm{~mL}$ of primary $\mathrm{W} / \mathrm{O}$ emulsion was gradually added to $10 \mathrm{~mL}$ of distilled water containing $5 \% \mathrm{w} / \mathrm{v}$ of Tween 20 at $750 \mathrm{rpm}$ for $10 \mathrm{~min}$ using High-speed Stirrer (Remi, India) to form multiple emulsion.

\section{Experimental design}

Preliminary screening using Plackett-Burman design (PBD): PBD was utilized for initial screening of significant variables affecting the particle size (PS) and entrapment efficiency (EE) of ME. The design consisted of 12 trials at 2 levels for 8 different variables using Minitab version 17 (Minitab Inc., USA, PA). The variables were (A) Concentration of lipophilic emulsifier in primary emulsion-W/O (5 and $15 \% \mathrm{w} / \mathrm{v}$ ), (B) Phase Volume Ratio (W:O) in primary emulsion (40:60 and 60:40), (C) Speed for Primary Emulsification (6500 and $9500 \mathrm{rpm}$ ), (D) Time for primary emulsification (3 and $7 \mathrm{~min}$ ), (E) Concentration of hydrophilic surfactant in secondary emulsion (3 and 7\%w/v), (F) Phase Volume Ratio (W/O : W) in secondary emulsion: (40:60 and 60:40), (G) Speed for secondary emulsification (500 and $1000 \mathrm{rpm}$ ) and (H) Time for secondary emulsification (5 and $15 \mathrm{~min}$ ). Relative effect of different variables were screened with the help of Pareto charts for observed responses [17].

Optimization of key variables via Box Behnken design (BBD): Based on results of the PBD, three most significant factors: Phase Volume Ratio in primary emulsion (X1), Time for primary emulsification (X2) and Concentration of hydrophilic surfactant in secondary emulsion (X3) that influenced PS (Y1) and EE (Y2) were studied using BBD. The coded and actual values of the variables in BBD are as shown in Table 1 . The BBD comprised of 17 runs, 3 -factors and 3-levels with five centre point trials for reproducibility. Second order polynomial models were generated with the results of BBD and were used for process optimization in preparation of ME with minimum PS and maximum EE [19].

Contour plots and response surface analysis: Two-dimensional contour plots were established between $\mathrm{X}_{1}$ and $\mathrm{X}_{2}, \mathrm{X}_{1}$ and $\mathrm{X}_{3}$, and $\mathrm{X}_{2}$ and $\mathrm{X}_{3}$ at constant level (0) of $\mathrm{X}_{3}, \mathrm{X}_{2}$ and $\mathrm{X}_{1}$, respectively for PS and EE.

Three dimensional response surface plots were established to study the interaction of two factors keeping other factor at fixed level.

Optimization and validation: The established contour plots, response surface plots and reduced polynomial equation were confirmed by performing check point analysis. Values of independent variables were taken from three check points on contour plots and the values of PS (Y1) and EE (Y2) were calculated by substituting the values in the reduced polynomial equation. MEs were prepared experimentally as explained earlier. Each batch was prepared three times and mean values were determined. Difference in the predicted

\begin{tabular}{|c|c|c|c|}
\hline \multirow[t]{2}{*}{ Factors: Independent variables } & \multicolumn{3}{|c|}{ Levels Used } \\
\hline & -1 & 0 & +1 \\
\hline X1=Phase Volume Ratio (Ratio of W: O) in primary emulsion & 4 & 5 & 6 \\
\hline $\mathrm{X} 2=$ Time for primary emulsification $(\mathrm{min})$ & 3 & 5 & 7 \\
\hline X3=Concentration of hydrophilic surfactant in secondary emulsion (\%) & 3 & 5 & 7 \\
\hline Responses & \multicolumn{3}{|c|}{ Constraints } \\
\hline Y1=Particle size $(\mu \mathrm{m})$ & \multicolumn{3}{|c|}{ Minimize } \\
\hline Y2=Entrapment efficiency (\%) & \multicolumn{3}{|c|}{ Maximize } \\
\hline
\end{tabular}

Table 1: Variables and their levels in the Box Behnken design. 
and actual values of experimentally obtained PS and EE were checked using student's ' $\mathrm{t}$ ' test.

Simultaneous optimization technique (Desirability function) was used to simultaneously optimize the PS and EE [20]. Design Expert software (version 8.0.3, Suite, Minneapolis, USA) was used to calculate total desirability. Criteria of minimum PS and maximum EE were set to predict optimum conditions.

\section{Characterization and Evaluation of ME}

\section{Macroscopic and microscopic evaluation}

Macroscopic evaluation was performed in order to judge the homogeneity of the ME formulations. Formulations were visually observed for homogeneity, color, consistency and appearance [10]. Optical microscope (Olympus BX 40, USA) connected with a camera was used for microscopic evaluation and observations were made at 40X magnification after suitable dilutions with distilled water.

\section{Globule size determination}

The mean globule size and size distribution of the ME were measured by dynamic laser light scattering technique using particle size analyzer (Malvern Mastersizer 2000, UK) [10]. Particle size measurements were carried out at a $90^{\circ}$ scattering angle. The samples were freshly diluted with distilled water before analysis. Each measurement was performed in triplicate.

\section{Zeta potential}

The zeta potential of the ME was determined by the laser doppler electrophoretic mobility measurement technique using Zetasizer (Malvern Nano ZS90, U.K.) at $25^{\circ} \mathrm{C}$. All the measurements were carried out in triplicate.

\section{Entrapment efficiency}

$5 \mathrm{~mL}$ of freshly prepared ME was centrifuged (Sigma Centrifuge - 3K30, Germany) at $4500 \mathrm{rpm}$ for $10 \mathrm{~min}$ at room temperature. The supernatant was collected, suitably diluted using DMF and analysed for free drug content spectrophotometrically at $595 \mathrm{~nm}$ (UV - 1800, Shimadzu, Japan) [21]. Free drug content was calculated using regression equation $(\mathrm{Y}=0.0135 \mathrm{X}+0.0211)$ obtained in concentration range of $10-50 \mu \mathrm{g} / \mathrm{ml}$. EE was calculated [10] by equation (1)

$$
\mathrm{EE} \%=\frac{\text { Total Drug Added }- \text { Free Drug }}{\text { Total Drug Added }} \times 100
$$

\section{Rheological analysis}

Rheological analysis was performed employing Brookfield viscometer (DV-I Prime, Brookfield, USA) using S61 spindle at 20 rpm at room temperature. All the measurements were carried out in triplicate. The rheological behaviour of each ME was evaluated by plotting the viscosity $(\mathrm{cP})$ versus shear rate $(1 / \mathrm{s})$.

\section{In vitro diffusion studies}

In vitro drug diffusion studies were carried out using dialysis bag technique [22]. Dialysis bags with a molecular weight cut-off of 12000 Daltons and pore size $2.4 \mathrm{~nm}$ (Hi-media, Mumbai, India) were washed with distilled water and soaked in $\mathrm{pH} 7.4$ phosphate buffer saline (PBS) overnight before use. $2 \mathrm{~mL}$ of freshly prepared MEs (equivalent to $1 \mathrm{mg} /$ $\mathrm{mL}$ of Lisinopril dihydrate) was introduced into the dialysis bag and was tightly sealed at each end with thread. The dialysis bag was placed over magnetic stirrer in a beaker containing $25 \mathrm{~mL}$ of PBS pH-7.4 at
$100 \mathrm{rpm}$ and temperature was maintained at $37 \pm 0.5^{\circ} \mathrm{C}$. Aliquots were withdrawn at $0.25,0.5,1,2,4,6$ and $8 \mathrm{~h}$ and replaced with fresh buffer solution in order to maintain sink conditions [23]. Diffusion of plain drug solution ( $1 \mathrm{mg} \mathrm{LD} / \mathrm{mL}$ in PBS $\mathrm{pH}-7.4$ buffer) was also studied in a similar manner. The samples were analysed spectrophotometrically at $595 \mathrm{~nm}$ after suitable dilutions. The studies were carried out three times and cumulative percentage drug release was calculated. In vitro release study data was further fitted to various release models viz zero order, first order, Korsemeyer Peppas and Higuchi model to identify the mechanism and kinetics of drug release from formulated MEs. The drug release at different time points was calculated using regression equation $(\mathrm{Y}=0.012 \mathrm{X}+0.077)$ obtained in concentration range of $10-50$ $\mu \mathrm{g} / \mathrm{ml}$. Regression coefficient $\left(\mathrm{r}^{2}\right)$ was calculated to identify the best-fit model [10].

\section{Ex vivo permeability studies}

The ex vivo permeability studies of LD loaded ME and plain drug solution were performed across rat intestine. The study protocols were approved by the Institutional Animal Ethics Committee of Pharmacy department, M S University of Baroda, Vadodara, India. For this study, non fasting male albino rats $(250-300 \mathrm{~g})$ were sacrificed by overdose of anaesthesia. Intestine was immediately isolated, washed properly with PBS pH 7.4 buffer solution and examined for integrity. The excised intestinal membrane was then mounted on Franz diffusion cell in such a manner that membrane did not shift from its place once the dosage form was placed onto it. The hooks were secured with rubber bands on the sides of both compartments to make it leak proof. Franz diffusion cell had a diameter of $16.9 \mathrm{~mm}$ and membrane with thickness of $0.2 \pm 0.1 \mathrm{~mm}$. The area available for diffusion was about $2.25 \mathrm{~cm}^{2}$. The temperature of the receiver chamber containing $20 \mathrm{~mL}$ of diffusion media (phosphate buffer, $\mathrm{pH}$ 7.4) was controlled at $37 \pm$ $0.5^{\circ} \mathrm{C}$ under continuous stirring with Teflon-coated magnetic bar at a constant rate, in a way that the membrane surface just flushes the diffusion fluid. $2 \mathrm{~mL}(1 \mathrm{mg} / \mathrm{mL})$ of MEs and plain drug solution was placed in the donor compartment of Franz diffusion cell. Samples were withdrawn and replenished by the same volume of fresh buffer solution at predetermined time intervals. The drug content was determined spectrophotometrically at $595 \mathrm{~nm}$ for estimation of LD after suitable dilutions with PBS [10,24-26].

The formulations were studied in triplicate for permeability studies. The cumulative amount of drug permeated $\left(\mu \mathrm{g} / \mathrm{cm}^{2}\right)$ was plotted as a function of time $(t)$ for each formulation. Drug flux (permeation rate) at steady state (Jss) was calculated by dividing the slope of the linear portion of the graph by the area of the diffusion cell. The diffusion coefficient was calculated by dividing Jss by the initial concentration of drug in the donor cell $\left(\mathrm{C}_{0}\right)$ and the mean cumulative values for \%drug diffused versus time were plotted against time. The slopes of the graphs were used to calculate the diffusion coefficients and the results were subjected to one-way ANOVA [26].

$$
\begin{aligned}
& \text { Enhancement ratio (Er) was calculated [25] by Equation (2) } \\
& \mathrm{Er}=\frac{\text { Jss of ME }}{\text { Jss of Plain Drug Solution }} \\
& \text { Where, Jss is drug flux at steady state. }
\end{aligned}
$$

\section{Stability studies}

The optimized formulations were subjected to stability testing as per International Conference on Harmonization (ICH) Q1A (R2) guidelines (ICH, 2003). Optimized formulations were sealed in Type-I transparent glass vials and stored at refrigerated condition $\left(2-8^{\circ} \mathrm{C}\right)$, 
Citation: Sawant KK, Mundada VP, Patel VJ (2017) Development and Optimization of w/o/w Multiple Emulsion of Lisinopril Dihydrate Using Plackett Burman and Box-Behnken Designs. J Nanomed Nanotechnol 8: 422. doi: 10.4172/2157-7439.1000422

long term condition $\left(25 \pm 2^{\circ} \mathrm{C} / 60 \pm 5 \% \mathrm{RH}\right)$, and accelerated condition $\left(40^{\circ} \mathrm{C} \pm 2^{\circ} \mathrm{C} / 75 \pm 5 \% \mathrm{RH}\right)$ in stability chamber (S. R. Lab Instruments, Mumbai, India) for a period of 3 months. Samples were also observed visually for phase separation and by microscopy for homogeneity (to confirm its multiple nature). At monthly intervals, samples were analysed for PS, zeta potential and EE. Each study was performed in triplicate.

\section{Statistical Analysis}

The experimental data were validated by Analysis of Variance (ANOVA) followed by F-test and Student $\mathrm{t}$-test which was determined at $95 \%(\alpha=0.05)$ significance level using Microsoft excel software (version: 2007). The co-efficient of multiple regression analysis $\left(\mathrm{R}^{2}\right)$ and the Fisher $\mathrm{F}$ test (Fisher variation ratio, the ratio of mean square for regression to mean square for residual) of the model were used in the statistical evaluation.

\section{Results and Discussion}

\section{Selection of oil}

Nature of oil is of great importance in case of ME since it controls the permeability of the liquid membrane, which in turn controls the release of solute across it [27]. To minimize the leaching of the drug to the external aqueous phase, the oil in which drug had minimum solubility was selected. Solubility of LD was measured in different oils to select the most appropriate oil for the preparation of MEs (Table 2). From the results obtained, it was observed that solubility of LD was minimum in corn oil when compared to other studied oils. Hence corn oil was selected to prepare the w/o/w ME of LD.

\begin{tabular}{|c|c|}
\hline Oil & Solubility $(\mathbf{m g} / \mathbf{m L} \pm \mathbf{S D})$ \\
\hline Liquid Paraffin light & $0.633 \pm 0.064$ \\
\hline Sunflower oil & $1.464 \pm 0.096$ \\
\hline Corn oil & $0.356 \pm 0.028$ \\
\hline Linseed oil & $0.426 \pm 0.067$ \\
\hline Soyabean oil & $1.234 \pm 0.088$ \\
\hline
\end{tabular}

Table 2: Solubility of LD in various oils

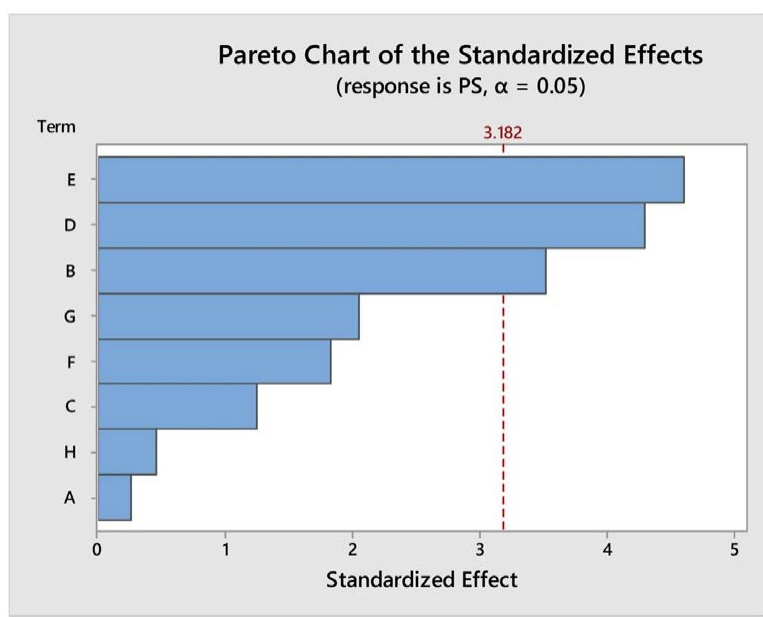

Figure 1: Pareto chart for Particle Size where $A$ is Concentration of lipophilic emulsifier in primary emulsion-W/O, B is Phase Volume Ratio (W: O) in primary emulsion, $C$ is Speed for Primary Emulsification, D is Time for primary emulsification, $E$ is Concentration of hydrophilic surfactant in secondary emulsion, $F$ is Phase Volume Ratio (W/O: W) in secondary emulsion, $G$ is Speed for secondary Emulsification, $\mathrm{H}$ is Time for secondary emulsification.

\section{Plackett-Burman design}

The PBD was used for initial screening and segregation of variables that significantly influenced the PS and EE of MEs. The relative effects of each factor are shown as Pareto charts in Figures 1 and 2.

As per Pareto charts, Concentration of hydrophilic surfactant in secondary emulsion, Phase Volume Ratio (Ratio of W:O) in primary emulsion and Time for primary emulsification were the variables that significantly $(\mathrm{P}<0.05)$ influenced both PS and EE of MEs. The PS varied from $15.30 \mu \mathrm{m}$ to $40.32 \mu \mathrm{m}$ and EE varied from $62.35 \%$ to $93.54 \%$ based on various factor combinations involved. The results obtained in Pareto charts can be observed visually using half normal plots [28]. Sometimes more than one factor seems to be almost significant (variable $\mathrm{F}$ in Figure 2) but when visualised in half normal plots, we get actual idea that such variables only act as a pseudo significant factor. Half-normal plots (Figures 3 and 4 ) gave a visual indication that factors D, E and $B$ had significant effect on PS and EE. Hence these three factors were taken for final optimization of the MEs by Box Behnken Design.

\section{Box Behnken design (BBD)}

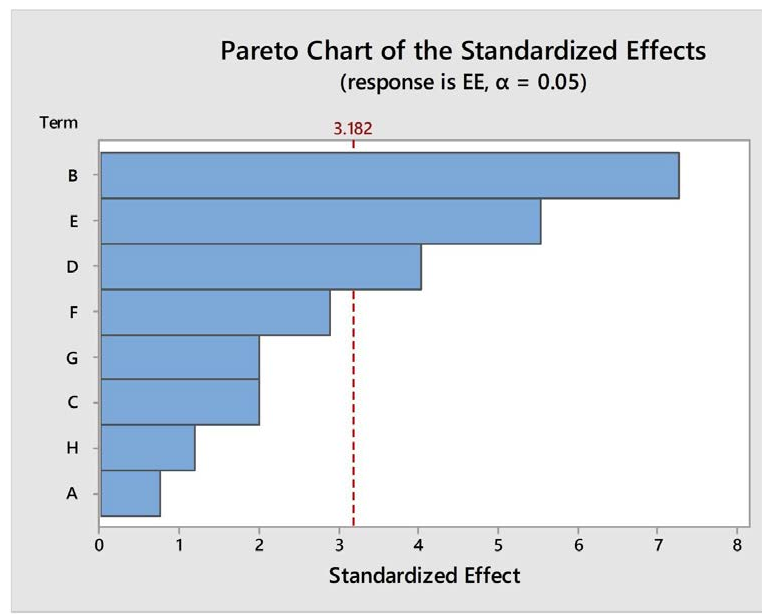

Figure 2: Pareto chart for Entrapment Efficiency where $A$ is Concentration of lipophilic emulsifier in primary emulsion-W/O, B is Phase Volume Ratio (W: O) in primary emulsion, $C$ is Speed for Primary Emulsification, D is Time for primary emulsification, $E$ is Concentration of hydrophilic surfactant in secondary emulsion, $F$ is Phase Volume Ratio (W/O: W) in secondary emulsion, G is Speed for secondary Emulsification, $\mathrm{H}$ is Time for secondary emulsification.

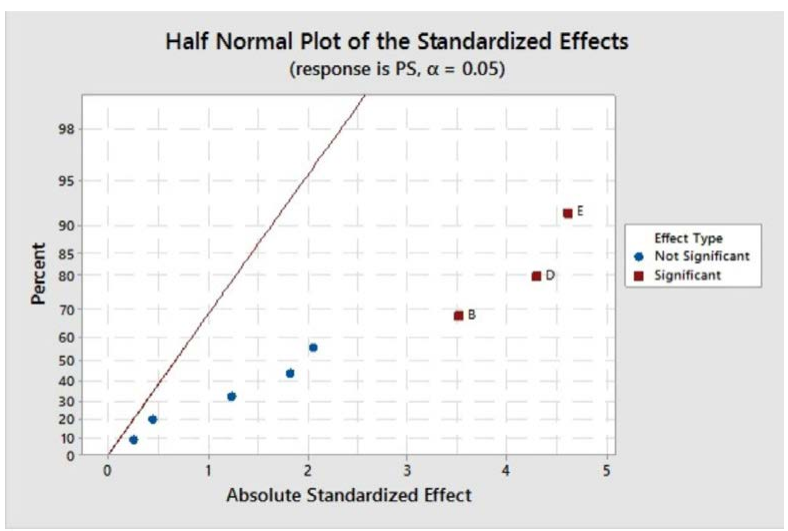

Figure 3: Half normal plot for particle size. 
Citation: Sawant KK, Mundada VP, Patel VJ (2017) Development and Optimization of w/o/w Multiple Emulsion of Lisinopril Dihydrate Using Plackett Burman and Box-Behnken Designs. J Nanomed Nanotechnol 8: 422. doi: 10.4172/2157-7439.1000422

Page 5 of 11

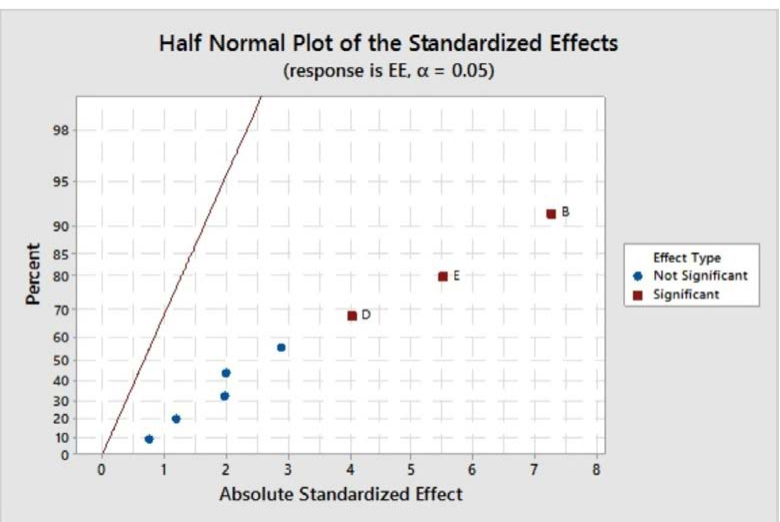

Figure 4: Half normal plot for entrapment efficiency.

\begin{tabular}{|c|c|c|c|c|c|}
\hline Batch. No. & $\mathbf{X}_{\mathbf{1}}$ & $\mathbf{X}_{2}$ & $\mathbf{X}_{3}$ & $\mathbf{Y}_{\mathbf{1}}(\mathbf{i n} \boldsymbol{\mu m})$ & $\mathbf{Y}_{\mathbf{2}}$ (in \%) \\
\hline BB1 & 0 & 0 & 0 & $17.74 \pm 0.99$ & $92.62 \pm 4.62$ \\
\hline BB2 & 1 & 1 & 0 & $33.98 \pm 1.65$ & $72.35 \pm 1.32$ \\
\hline BB3 & -1 & 0 & 1 & $23.62 \pm 3.42$ & $85.25 \pm 5.85$ \\
\hline BB4 & 1 & 0 & -1 & $25.96 \pm 1.74$ & $77.36 \pm 2.47$ \\
\hline BB5 & -1 & 1 & 0 & $27.02 \pm 2.44$ & $84.41 \pm 3.36$ \\
\hline BB6 & -1 & 0 & -1 & $19.54 \pm 0.95$ & $85.87 \pm 2.14$ \\
\hline BB7 & 0 & 0 & 0 & $16.74 \pm 2.12$ & $92.62 \pm 5.68$ \\
\hline BB8 & 1 & -1 & 0 & $33.54 \pm 1.05$ & $77.54 \pm 1.42$ \\
\hline BB9 & -1 & -1 & 0 & $27.56 \pm 1.74$ & $83.98 \pm 5.89$ \\
\hline BB10 & 0 & 1 & 1 & $36.56 \pm 0.95$ & $71.65 \pm 3.69$ \\
\hline BB11 & 0 & -1 & -1 & $32.98 \pm 3.70$ & $74.21 \pm 6.84$ \\
\hline BB12 & 0 & -1 & -1 & $34.68 \pm 1.25$ & $74.65 \pm 5.74$ \\
\hline BB13 & 0 & 0 & 0 & $16.74 \pm 1.89$ & $92.62 \pm 2.89$ \\
\hline BB14 & 0 & 0 & 0 & $15.54 \pm 2.36$ & $93.62 \pm 4.95$ \\
\hline BB15 & 1 & 0 & 1 & $29.24 \pm 0.98$ & $75.35 \pm 3.32$ \\
\hline BB16 & 0 & -1 & 1 & $32.85 \pm 2.11$ & $74.84 \pm 6.84$ \\
\hline BB17 & 0 & 0 & 0 & $16.74 \pm 1.65$ & $92.62 \pm 4.54$ \\
\hline
\end{tabular}

Table 3: Design matrix for BBD for Lisinopril dihydrate MEs.

Design Expert software was extensively used for designing the sequence of trials and interpreting the results [17]. BBD is rotatable or nearly rotatable second-order design based on three-level incomplete factorial design [29]. Using Box Behnken Design, seventeen batches of LD MEs were prepared by varying three independent variables, Phase Volume Ratio (W: O) in primary emulsion $\left(\mathrm{X}_{1}\right)$, Time for primary emulsification $(\mathrm{min})\left(\mathrm{X}_{2}\right)$, and Concentration of hydrophilic surfactant in secondary emulsion $(\%)\left(\mathrm{X}_{3}\right)$. PS $\left(\mathrm{Y}_{1}\right)$ and EE $\left(\mathrm{Y}_{2}\right)$ were taken as the dependent variables and the results were recorded. The PS and EE values for the 17 batches showed a wide variation from 16.74 to 36.56 $\mu \mathrm{m}$ and 71.65 to $93.62 \%$, respectively (Table 3 ).

This variation is reflected in equation 3 and equation 4 for PS and EE respectively. The $\mathrm{p}$-value and $\mathrm{t}$-stat demonstrated the significance of each coefficient. The corresponding coefficient is found to be more significant when smaller is the $\mathrm{p}$ value and larger is the magnitude of the ' $t$ ' value (Table 4) [30].

$$
\begin{aligned}
& Y_{1}=16.70+3.12 X_{1}+0.66 X_{2}+1.14 X_{3}+0.24 X_{1} X_{2}-0.20 X_{1} X_{3}+ \\
& 0.50 X_{2} X_{3}+2.07 X_{1}^{2}+11.7 X_{2}^{2}+5.82 X_{3}^{2} \\
& Y_{2}=92.82-4.61 X_{1}-0.94 X_{2}-0.63 X_{3}-1.41 X_{1} X_{2}-0.35 X_{1} X_{3}- \\
& 0.91 X_{2} X_{3}-3.07 X_{1}^{2}-10.19 X_{2}^{2}-8.80 X_{3}^{2}
\end{aligned}
$$

The quadratic model was significant for both PS and EE with F-value of 67.37 and 632.30 respectively. Value of regression coefficient $\left(\mathrm{R}^{2}=0.9886\right.$ for $\mathrm{PS}$ and $\left.\mathrm{R}^{2}=0.9988\right)$ signified good correlation between the responses and the chosen variables.

For PS, coefficients $\mathrm{X}_{1}, \mathrm{X}_{3}, \mathrm{X}_{1}^{2,} \mathrm{X}_{2}^{2} \mathrm{X}_{3}^{2}$ were significant while $\mathrm{X}_{2}, \mathrm{X}_{1}$ $\mathrm{X}_{2}, \mathrm{X}_{1} \mathrm{X}_{3}, \mathrm{X}_{2} \mathrm{X}_{3}$ were least contributing (Equation 3) and therefore were neglected from the full model. Since there were many insignificant terms, model reduction was used to simplify the model. Reduced polynomial equation obtained after model reduction is shown in Equation 5. After model reduction, the "Pred R-Squared" of 0.9559 was in reasonable agreement with the "Adj R-Squared" of 0.9754.

$$
Y_{1}=16.70+3.12 X_{1}+1.14 X_{3}+2.07 X_{1}^{2}+11.75 X_{2}^{2}+5.82 X_{3}^{2}
$$

Similarly, For EE, coefficients $\mathrm{X}_{1}, \mathrm{X}_{2} \mathrm{X}_{3}, \mathrm{X}_{1}^{2,} \mathrm{X}_{2}^{2} \mathrm{X}_{3}^{2} \mathrm{X}_{1} \mathrm{X}_{2}, \mathrm{X}_{2} \mathrm{X}_{3}$ were significant while only $X_{1} X_{3}$ was least contributing (Equation 4 ) and therefore neglected from the full model. Reduced polynomial equation obtained after model reduction was represented as Equation 6. After model reduction, the "Pred R-Squared" of 0.9894 was in reasonable agreement with the "Adj R-Squared" of 0.9969.

$$
\begin{aligned}
& Y_{2}=92.82-4.61 X_{1}-0.94 X_{2}-0.63 X_{3}-1.41 X_{1} X_{2}- \\
& 0.91 X_{2} X_{3}-3.07 X_{1}^{2}-10.19 X_{2}^{2}-8.80 X_{3}^{2}
\end{aligned}
$$

\begin{tabular}{|c|c|c|c|c|c|c|}
\hline \multirow[t]{2}{*}{ Source } & \multicolumn{2}{|c|}{ PS } & \multirow[t]{2}{*}{ t-stat } & \multicolumn{2}{|c|}{ EE } & \multirow[t]{2}{*}{ t-stat } \\
\hline & Coefficients & p-value & & Coefficients & p-value & \\
\hline Model & - & $<0.0001$ & & - & $<0.0001$ & \\
\hline Intercept & 16.70 & 9.73E-09 & 30.8410 & 92.82 & $4.57 E-17$ & 478.9602 \\
\hline $\mathrm{X}_{1}$ & 3.12 & 0.0002 & 7.2941 & -4.61 & $<0.0001$ & -30.1142 \\
\hline$x_{2}$ & 0.66 & 0.1650 & 1.5505 & -0.94 & 0.0005 & -6.12728 \\
\hline$x_{3}$ & 1.14 & 0.0325 & 2.6601 & -0.63 & 0.0047 & -4.07941 \\
\hline$X_{1} X_{2}$ & 0.24 & 0.6978 & 0.4047 & -1.41 & 0.0003 & -6.48454 \\
\hline$x_{1} X_{3}$ & -0.20 & 0.7508 & -0.3304 & -0.35 & 0.1528 & -1.60383 \\
\hline $\mathrm{X}_{2} \mathrm{X}_{3}$ & 0.50 & 0.4339 & 0.8300 & -0.91 & 0.0041 & -4.18841 \\
\hline $\mathrm{X}_{1}^{2}$ & 2.07 & 0.0098 & 3.5144 & -3.07 & $<0.0001$ & -14.5135 \\
\hline $\mathrm{X}_{2}^{2}$ & 11.7 & $<0.0001$ & 19.9150 & -10.19 & $<0.0001$ & -48.2283 \\
\hline $\mathrm{X}_{3}^{2}$ & 5.82 & $<0.0001$ & 9.8569 & -8.80 & $<0.0001$ & -41.6582 \\
\hline $\begin{array}{l}\text { Residual: } \\
\text { Lack of fit }\end{array}$ & - & 0.0966 & & - & 0.5316 & \\
\hline
\end{tabular}

The results of ANOVA of the second order polynomial equation of PS and EE are given in Tables 5 and 6 , respectively. F tabulated was found to be higher than F calculated for both PS and EE. Therefore, the neglected values did not significantly contribute for the prediction of responses selected [31]. Hence F-statistic justified the reduction of

Significant terms having $p$-value $<0.05$ were represented in italics.

Table 4: Regression Coefficients and their p-values for PS and EE.

\begin{tabular}{|c|c|c|c|c|c|c|c|}
\hline \multicolumn{2}{|c|}{} & df & SS & MS & $\mathbf{F}$ & $\mathbf{R}^{\mathbf{2}}$ & Adjusted $\mathbf{R}^{2}$ \\
\hline \multirow{2}{*}{ Regression } & FM & 9 & 888.96 & 98.77 & 67.37 & 0.9886 & 0.9739 \\
\cline { 2 - 8 } & RM & 5 & 884.03 & 176.81 & 127.98 & 0.9831 & 0.9754 \\
\hline \multirow{2}{*}{$\begin{array}{c}\text { Residual } \\
\text { (Error) }\end{array}$} & FM & 7 & 10.26 & 1.47 & & & \\
\cline { 2 - 8 } & RM & 11 & 15.20 & 1.38 & & & \\
\hline
\end{tabular}

Notes: SS of Error $_{(\mathrm{RM})}-\mathrm{SS}$ of Error $_{(\mathrm{FM})}=15.20-10.26=4.94$.

No. of parameters omitted $=4$.

MS of error $=1.47$

$\mathrm{F}$ calculated $=\left[\left(\mathrm{SS}\right.\right.$ of Error $_{(\mathrm{RM})}-\mathrm{SS}$ of Error $\left._{(\mathrm{FM})}\right) / \mathrm{No}$. of parameters omitted $] / \mathrm{MS}$ of error

$=(4.94 / 4) / 1.47$

$=0.840$

$F$ tabulated $($ at $p<0.05)=4.12$

Table 5: Analysis of variance (ANOVA) of full model (FM) and reduced model (RM) for PS of ME of LD. 
Citation: Sawant KK, Mundada VP, Patel VJ (2017) Development and Optimization of w/o/w Multiple Emulsion of Lisinopril Dihydrate Using Plackett Burman and Box-Behnken Designs. J Nanomed Nanotechnol 8: 422. doi: 10.4172/2157-7439.1000422

Page 6 of 11

models.

\section{Contour plots and response surface analysis}

For each PS and EE, Contour plots were generated between $\mathrm{X}_{1}$ VS. $\mathrm{X}_{2}, \mathrm{X}_{1}$ VS. $\mathrm{X}_{3}$, and $\mathrm{X}_{2}$ VS. $\mathrm{X}_{3}$ at fixed level (0) of third variable as shown in Figures 5 and 6, respectively. For PS (Figure 5A and 5B), the plot formed parabolic shape and showed that increase in Phase Volume

\begin{tabular}{|c|c|c|c|c|c|c|c|}
\hline \multicolumn{2}{|c|}{} & df & SS & MS & F & $\mathbf{R}^{\mathbf{2}}$ & Adjusted R \\
\hline \multirow{2}{*}{ Regression } & FM & 9 & 1068.61 & 118.73 & 632.30 & 0.9988 & 0.9972 \\
\cline { 2 - 8 } & RM & 8 & 1068.13 & 133.52 & 594.23 & 0.9983 & 0.9966 \\
\hline \multirow{2}{*}{$\begin{array}{c}\text { Residual } \\
\text { (Error) }\end{array}$} & FM & 7 & 1.31 & 0.19 & & & \\
\cline { 2 - 8 } & RM & 8 & 1.80 & 0.22 & & & \\
\hline
\end{tabular}

Notes: SS of Error $_{(\mathrm{RM})}-\mathrm{SS}$ of Error $_{(\mathrm{FM})}=15.20-10.26=4.94$

No. of parameters omitted $=4$.

MS of error $_{(\mathrm{FM})}=1.47$.

$\mathrm{F}$ calculated $=\left[\left(\mathrm{SS}\right.\right.$ of Error $_{(\mathrm{RM})}-\mathrm{SS}$ of Error $\left._{(\mathrm{FM})}\right) / \mathrm{No}$. of parameters omitted]/ MS

of error

$=(4.94 / 4) / 1.47$

$=0.840$

$F$ tabulated $($ at $p<0.05)=4.12$

Table 6: Analysis of variance (ANOVA) of full and reduced models for EE of ME of LD.
Ratio (W:O) in primary emulsion $\left(\mathrm{X}_{1}\right)$, increased particle size whereas time for primary emulsification $\left(\mathrm{X}_{2}\right)$ and Concentration of hydrophilic surfactant in secondary emulsion $\left(\mathrm{X}_{3}\right)$ gave minimum particle size when these variables has mid values of $5 \mathrm{~min}$ and $5 \%$ respectively when plotted against $\mathrm{X}_{1}$. On either side of mid value, particle size increased. Lowest PS of $15.65 \mu \mathrm{m}$ was observed at lowest value of $\mathrm{X}_{1}$. However, the Contour of Time for primary emulsification $\left(\mathrm{X}_{2}\right)$ VS. Concentration of hydrophilic surfactant in secondary emulsion $\left(\mathrm{X}_{3}\right)$ (Figure $5 \mathrm{C}$ ) confirmed the minimum PS at the mid value.

For EE (Figure 6A), maximum entrapment was observed at low level of Phase Volume Ratio (W:O) in primary emulsion $\left(\mathrm{X}_{1}\right)$. However, Concentration of hydrophilic surfactant in secondary emulsion $\left(\mathrm{X}_{3}\right)$ should be in middle range to achieve maximum EE (Figure 6B) when plotted against $\mathrm{X}_{1}$. Highest $\mathrm{EE}$ of $94.37 \%$ was observed at lowest value of $\mathrm{X}_{1}$. The Contour of Time for primary emulsification $\left(\mathrm{X}_{2}\right)$ VS Concentration of hydrophilic surfactant in secondary emulsion $\left(\mathrm{X}_{3}\right)$ (Figure 6C) confirmed maximum EE at the mid value. Hence, it was concluded from the Contours that low Phase Volume Ratio (W:O) in primary emulsion (40:60), mid ranges of Time for primary emulsification (5 min) and Concentration of hydrophilic surfactant in secondary emulsion (5\%) were required to attain minimum PS and maximum EE.
A

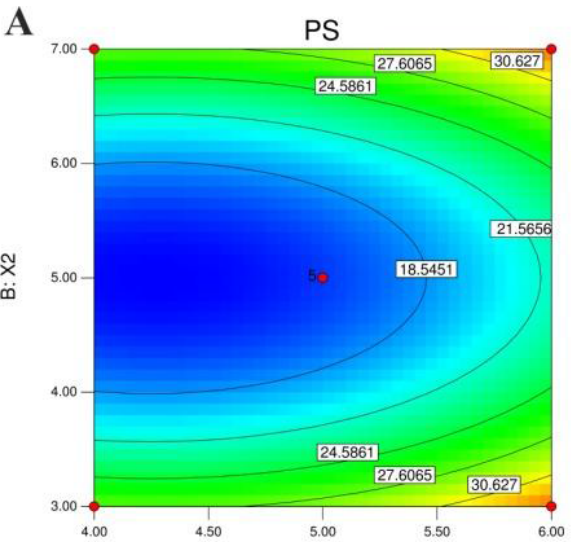

$\mathrm{A}: \mathrm{X} 1$
B

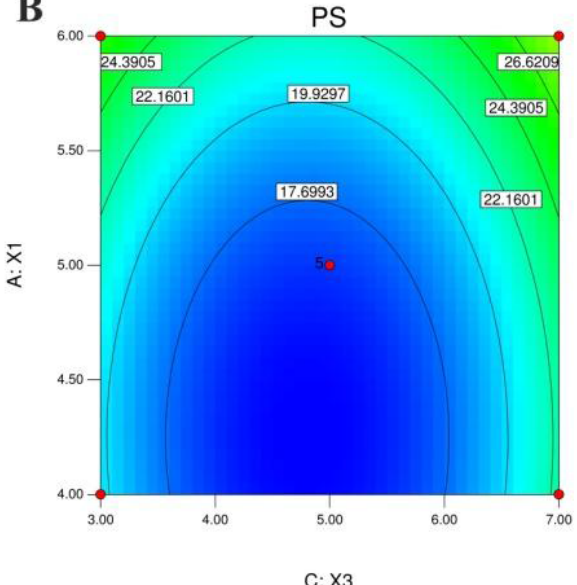

C

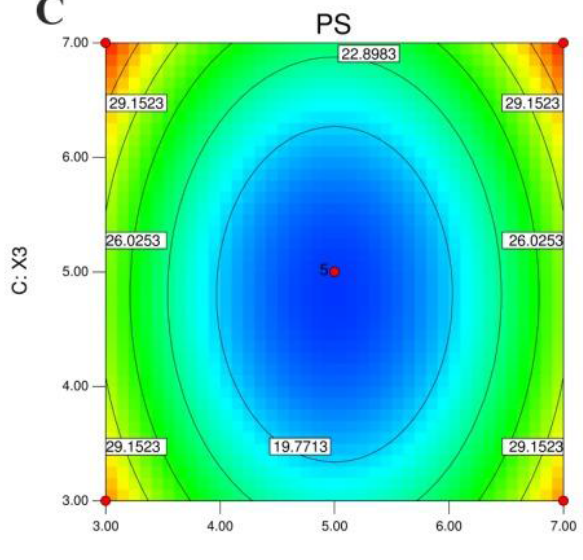

B: $\mathrm{X} 2$

Figure 5: Contour plots showing effect of (A) X1 vs X2 (at 0 level of $X 3$ ), (b) X1 vs X3 (at 0 level of $X 2$ ), and (c) X2 vs X3 (at 0 level of $X 1$ ) on $P S$ of $M E s$.
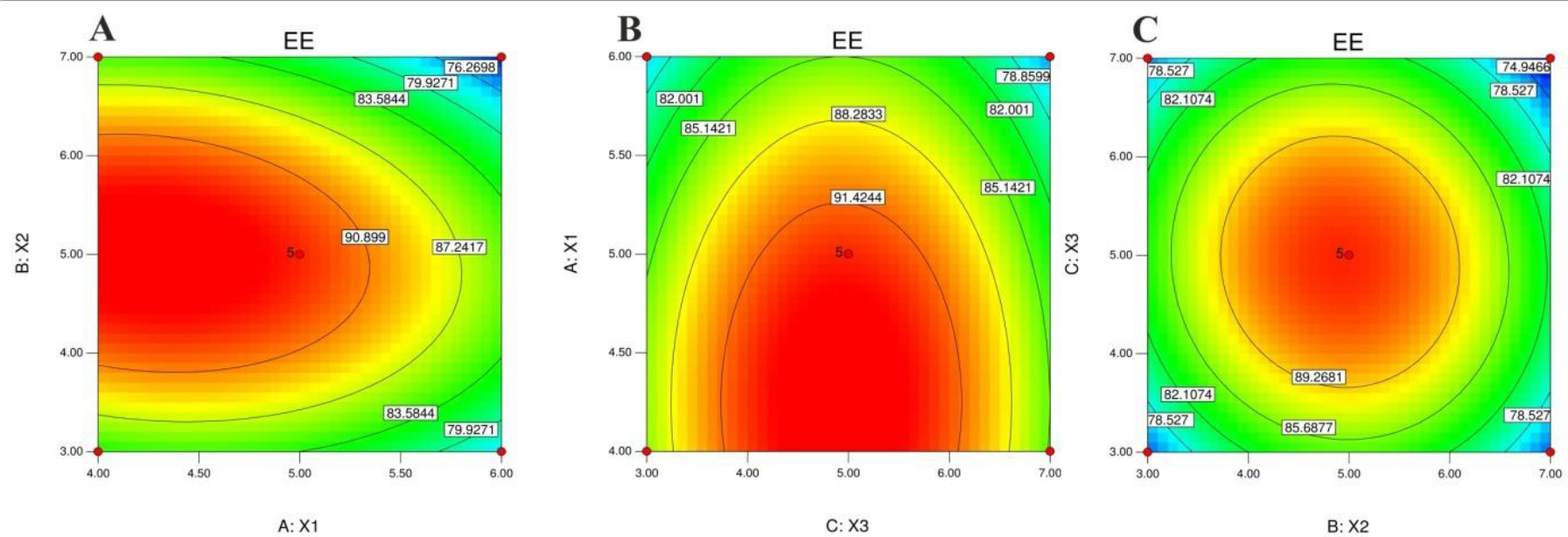

Figure 6: Contour plots showing effect of (A) X1 vs X2 (at 0 level of $X 3$ ), (b) X1 vs X3 (at 0 level of X2), and (c) X2 vs X3 (at 0 level of $X 1$ ) on EE of MEs. 
Citation: Sawant KK, Mundada VP, Patel VJ (2017) Development and Optimization of w/o/w Multiple Emulsion of Lisinopril Dihydrate Using Plackett Burman and Box-Behnken Designs. J Nanomed Nanotechnol 8: 422. doi: 10.4172/2157-7439.1000422

Response surface plots show the relationship between these variables even more clearly when plotted between $\mathrm{X}_{1}$ and $\mathrm{X}_{2}, \mathrm{X}_{1}$ and $\mathrm{X}_{3}$, and $\mathrm{X}_{2}$ and $\mathrm{X}_{3}$ at constant level (0) of $\mathrm{X}_{3}, \mathrm{X}_{2}$ and $\mathrm{X}_{1}$, respectively for PS and EE [14]. Minimum PS and maximum EE were achieved with low level of Phase Volume Ratio (W:O) in primary emulsion, middle range of Time for primary emulsification and Concentration of hydrophilic surfactant in secondary emulsion (Figures 7 and 8 ).

\section{Optimization and validation}

The optimum formulation was selected based on the criteria of attaining the minimum value of PS and the maximum value of $\mathrm{EE}$. Priority levels were set as ' +++ ' for both PS and EE. The desirability function is a transformation of the response variable to a 0 to 1 scale. Response of 0 represents a completely undesirable response and 1 represents the most desirable response [32]. Based on this, thirty different solutions were predicted with the desirability of 1 .

Out of them, three check point formulations were selected, and the experimental and predicted results were compared. Data analysis using student's t-test showed that there was no statistically significant difference $(\mathrm{p}<0.05)$ between experimentally obtained values and predicted values (Table 7). Experimental values were found to be in close proximity to the predicted values and the low values of standard deviations confirmed the reproducibility of the results.

\section{Characterization and evaluation of $\mathrm{ME}$}

Based on the results of optimization, Span 80 and Span 83 were tried as the lipophilic surfactant at $10 \%$ and $20 \%$ concentrations, using $5 \%$ Tween 20 as the hydrophilic surfactant. The characteristics of different MEs like\%EE, droplet size, centrifugation stability and Zeta Potential were measured immediately after preparation as shown in Table 8.

Macroscopic and microscopic evaluation: In visual appearance, all formulations were homogeneous and white in color with very good consistency. Optical image (Figure 9) clearly showed the multiple nature of the ME. Microscopic analysis revealed that many small droplets were present in the internal phase of the multiple globules indicating Type $\mathrm{B}$ w/o/w ME, as per the Florence and Whitehill classification [2].

Globule size determination: Globule size of optimized batches were found to be in the range of $15.65 \pm 1.97 \mu \mathrm{m}$ (PDI-0.214 \pm 0.098 ) to $20.21 \pm 2.23 \mu \mathrm{m}$ (PDI $-0.274 \pm 0.052$ ). MEs had narrow size distribution as PDI was in the range of $0.2-0.3$. From the results obtained, it was found that MEs prepared with Span 80 had slightly larger size as compared to MEs prepared with Span 83. The possible reason could be the difference in HLB values of Span 80 and Span 83. The lower particle size obtained with Span 83 may be attributed to its higher lipophilicity (HLB value 3.7$)$ and lower Critical Micelle Concentration (CMC) $(0.216 \mathrm{mM})$ as compared to Span 80 (HLB value 4.3 and CMC $0.43 \mathrm{mM}$ ) [10]. Increase in globule size might be due to higher accumulation of the surfactant at the interface resulting in a thicker interfacial film and hence increase in the globule size [33].

Zeta potential analysis: The zeta potential is indicative of the charge on the surface of the particles and a higher zeta potential value
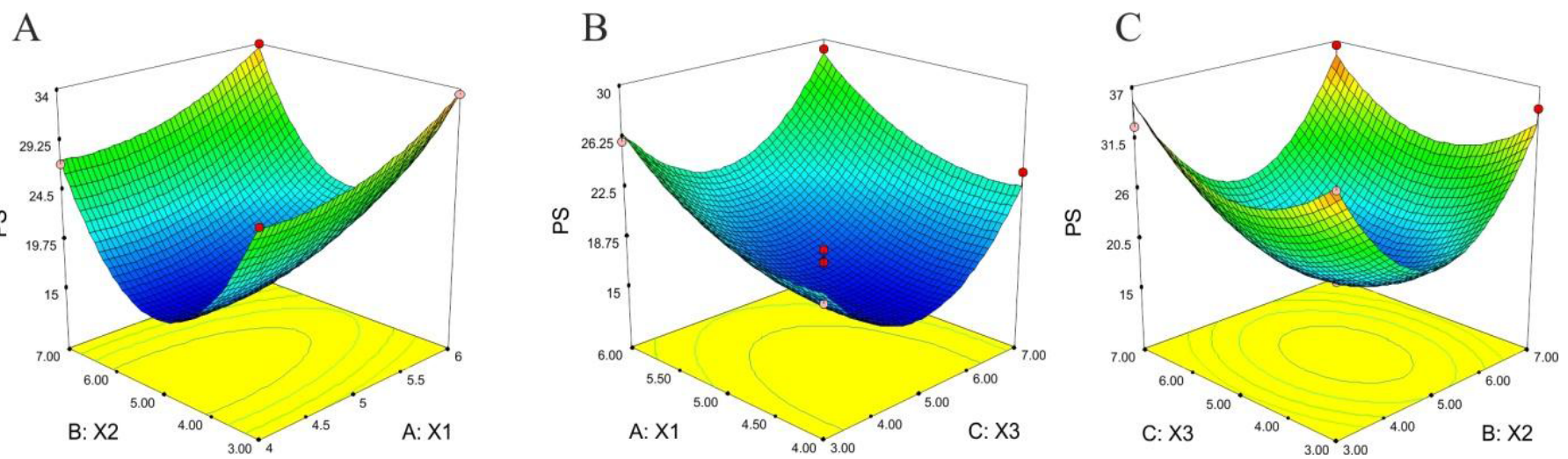

Figure 7: Response surface plot showing effect of (A) X1 vs X2 (at 0 level of X3), (b) X1 vs X3 (at 0 level of X2), and (c) X2 vs X3 (at 0 level of X1) on PS of MEs.

A

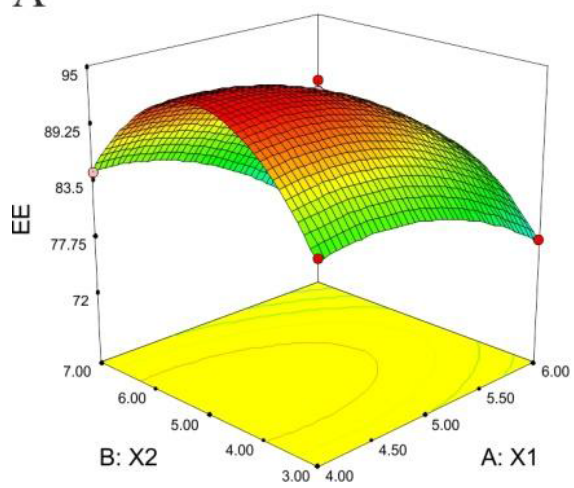

B

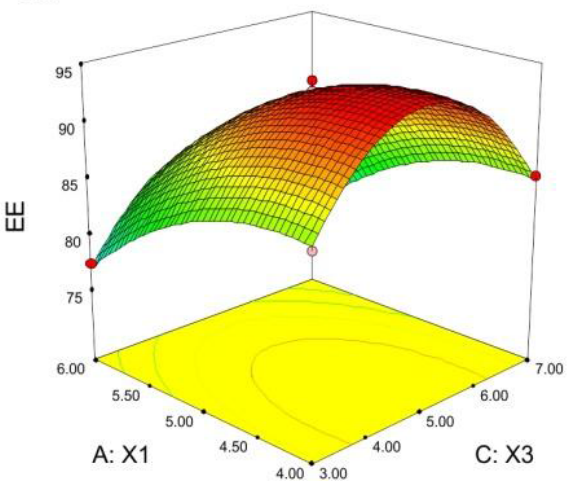

C

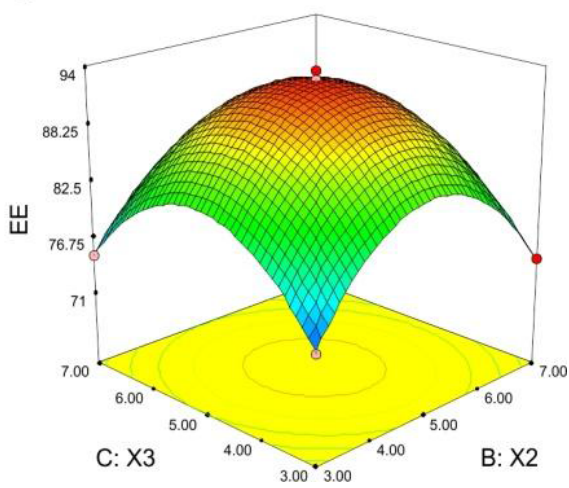

Figure 8: Response surface plot showing effect of (A) X1 vs X2 (at 0 level of $X 3$ ), (b) $X 1$ vs $X 3$ (at 0 level of $X 2$ ), and (c) X2 vs $X 3$ (at 0 level of $X 1$ ) on EE of MEs. 
Citation: Sawant KK, Mundada VP, Patel VJ (2017) Development and Optimization of w/o/w Multiple Emulsion of Lisinopril Dihydrate Using Plackett Burman and Box-Behnken Designs. J Nanomed Nanotechnol 8: 422. doi: 10.4172/2157-7439.1000422

Page 8 of 11

\begin{tabular}{|c|c|c|c|c|c|c|c|}
\hline \multicolumn{8}{|c|}{ Checkpoint batches with their experimental and predicted values of PS and EE } \\
\hline \multirow{2}{*}{ Batch No. } & \multirow[t]{2}{*}{$x_{1}$} & \multirow[t]{2}{*}{$X_{2}(\min )$} & \multirow[t]{2}{*}{$\mathrm{X}_{3}(\%)$} & \multicolumn{2}{|c|}{ PS $(\mu \mathrm{m})$} & \multicolumn{2}{|c|}{ EE (\%) } \\
\hline & & & & Experimental (Mean) & Predicted & Experimental (Mean) & Predicted \\
\hline 1 & $-0.7(4.30)$ & $0.055(5.11)$ & $-0.125(4.75)$ & 16.864 & 15.514 & 92.548 & 94.468 \\
\hline 2 & $-0.75(4.25)$ & $-0.035(4.93)$ & $-0.10(4.80)$ & 13.987 & 15.485 & 95.264 & 94.509 \\
\hline 3 & $-0.82(4.18)$ & $0.010(5.02)$ & $-0.45(4.71)$ & 14.541 & 15.492 & 93.745 & 94.454 \\
\hline \multicolumn{4}{|l|}{$t_{\text {calculated }}$} & \multicolumn{2}{|c|}{0.7154} & \multicolumn{2}{|c|}{0.5040} \\
\hline \multicolumn{4}{|c|}{ t } & \multicolumn{2}{|c|}{4.3026} & \multicolumn{2}{|c|}{4.3026} \\
\hline
\end{tabular}

Table 7: Check point analysis with 't' test analysis.

\begin{tabular}{|c|c|c|c|c|}
\hline Formulation & ME 1 (10\% Span 80$)$ & ME 2 (20\% Span 80) & ME 3 (10\% Span 83$)$ & ME 4 (20\% Span 83) \\
\hline Physical Appearance & +++ & +++ & +++ & +++ \\
\hline Multiple nature & +++ & +++ & +++ & +++ \\
\hline EE (\%) ${ }^{*}$ & $79.63 \pm 3.15$ & $72.35 \pm 2.98$ & $87.35 \pm 3.79$ & $80.26 \pm 3.48$ \\
\hline Droplet size $^{*}(\mu \mathrm{m})$ & $19.25 \pm 2.105$ & $20.21 \pm 2.226$ & $15.65 \pm 1.967$ & $17.06 \pm 2.112$ \\
\hline Centrifugation Stability & Stable & Stable & Stable & Stable \\
\hline Zeta Potential (mV) & $-16.10 \pm 1.14$ & $-18.21 \pm 2.01$ & $-15 \pm 2.33$ & $-17.84 \pm 3.58$ \\
\hline
\end{tabular}

+ Not satisfactory; ++ Good; +++ Very good.

*Value represented as the mean of three experiments \pm SD.

Table 8: Characteristics of different ME formulations.

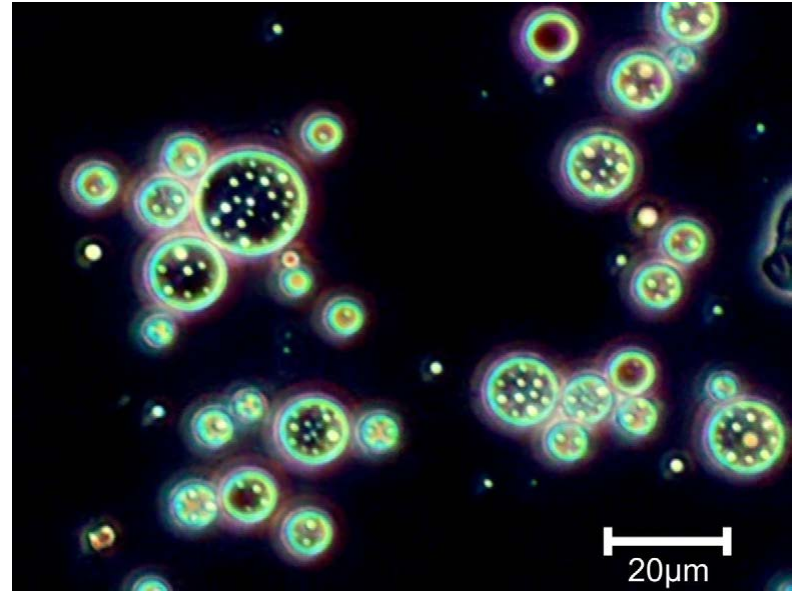

Figure 9: Optical photographs of w/o/w MEs (Under $40 \mathrm{X}$ ).

ensures that there is no phase separation, while a value closer to zero leads to risk of coalescence, thus suggests instability [15]. The results are shown in Table 8.

With increase in the concentration of the surfactant, there was decrease in zeta potential. This is possibly because the surfactant is nonionic and with increase in its concentration, total charge on the globule decreases due to increased surfactant coating which also results in increased PS [34]. Negative values of the zeta potential indicate that the electrostatic repulsion between globules will prevent their aggregation and thereby stabilize the MEs [35-37].

Entrapment efficiency: From the results (Table 8), the MEs prepared with Span 83 had higher EE as compared to MEs prepared with Span 80. Lower EE could be attributed to higher solubilisation effect produced by higher concentration of Span 83. At higher concentration of surfactant, solubility of LD in the external phase may increase due to diffusion of drug from lipid into aqueous phase leading to reduced EE [38].

Rheological analysis: All the MEs exhibited Non-Newtonian, shear thinning pseudoplastic flow behaviour, with viscosity of the systems

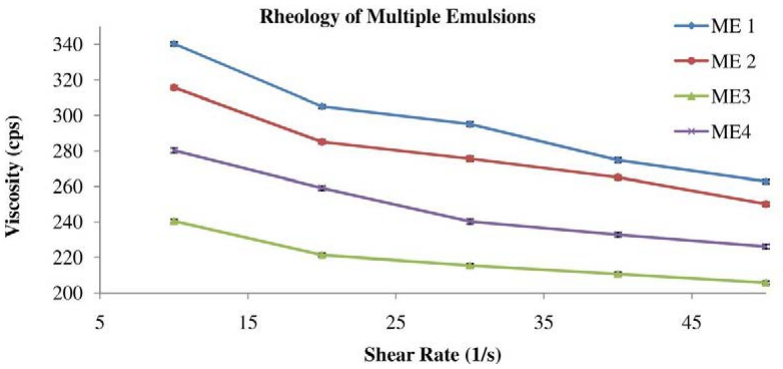

Figure 10: Graphical representation of Rheology of MEs.

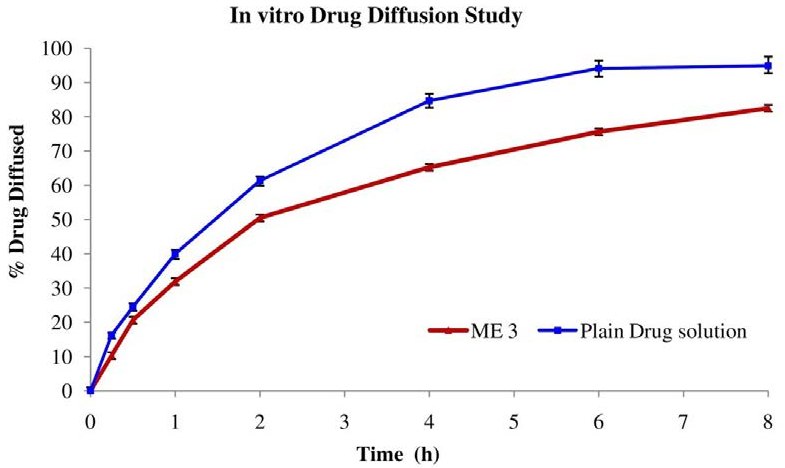

Figure 11: Release profiles of ME and plain drug solution through dialysis bag

decreasing with increasing shear rate (Figure 10). Such flow behavior is characteristic of very concentrated emulsions, with a volume fraction $\Phi \gg 0.74$ [39]. As a result, prepared MEs showed shear-thinning behavior [40] and the apparent viscosity decreased with increase in shear rate [41]. Furthermore, it was observed that Span 80 based MEs (ME 1 and ME 2) were more viscous as compared to Span 83 based MEs (ME 3 and ME 4). This again may be attributed to the lower HLB and greater micellization of Span 83 as compared to Span 80 [10]. Viscosity was found to increase with increase in concentration of Span.

Samples were also observed for any breakdown or loss in the integrity of multiple globules and phase inversion after viscosity 
measurements by optical microscopy. There was no destruction of multiple globules or phase inversion. It was concluded that shear stress used during rheological study did not induce structural changes in MEs, such as coalescence or phase inversion [23].

In vitro diffusion studies: The release profiles of $\mathrm{LD}$ from $\mathrm{ME}$ and plain drug solution through dialysis bag are presented in Figure 11. About $60 \%$ of drug was released in 2 hours and $94 \%$ of drug released in 6 hours from plain drug solution. MEs exhibited biphasic release with an initial rapid release phase (40-45\%) in $1^{\text {st }}$ two hours and then a slower release $(60-80 \%)$ between $2^{\text {nd }}$ to $8^{\text {th }}$ hour. Initial release of LD might be due to presence of $\mathrm{LD}$ in the external aqueous phase while the second prolonged release phase can be attributed to the slow release from the inner aqueous phase governed by the interfacial barrier of the oil phase [8]. In w/o/w ME, the drug in the internal phase is forced to partition itself through two phases prior to its release into the sink solution. The rate of release from internal aqueous phase into bulk will be governed by the nature of hydrophobic barrier, its thickness and any interaction between emulsifier and drug molecules [10]. The data (Table 9) obtained from in vitro drug release studies was fitted to various release models. The regression coefficient value was found to be highest $\left(r^{2}=0.9897\right)$ for Higuchi model [42]. The Higuchi model applied to a slab geometry permits definition of a release fraction linearly dependent on the square root of time and the reciprocal of the initial drug concentration. However, diffusion of LD through the oil film, is controlled by diffusion gradient regulation [43]. Similar work has been reported by Magdassi and Garti where they applied this model to multiple $\mathrm{W} / \mathrm{O} / \mathrm{W}$ emulsions taking into account the spherical geometry of the oil droplets [44]. Hence, it can be concluded that the release of LD from ME was by Higuchi diffusion based mechanism.

Ex vivo permeability studies: The permeation of $L D$ from $M E$ and plain drug solution through rat intestine is presented in Figure 12. The results indicate that diffusion was faster across the intestine from the ME as compared to the drug solution.

Drug flux (permeation rate) at steady state was found to be $119.3 \mu \mathrm{g} /$ $\mathrm{cm}^{2} / \mathrm{h}$ for ME and $105.1 \mu \mathrm{g} / \mathrm{cm}^{2} / \mathrm{h}$ for plain drug solution. Enhancement

\begin{tabular}{|c|c|c|c|c|}
\hline \multirow{2}{*}{$\begin{array}{c}\text { In vitro release } \\
\text { study }\end{array}$} & \multicolumn{4}{|c|}{ Linear Correlation Coefficient $\left(\mathbf{r}^{2}\right)$ Values } \\
\cline { 2 - 5 } & $\begin{array}{c}\text { Zero } \\
\text { order }\end{array}$ & First order & Higuchi & $\begin{array}{c}\text { Korsemeyer } \\
\text { Peppas }\end{array}$ \\
\cline { 2 - 5 } & 0.9310 & 0.9722 & 0.9897 & 0.8562 \\
\hline
\end{tabular}

Table 9: Linear correlation coefficient values of various models for in vitro release study.

Ex vivo Drug Diffusion Study

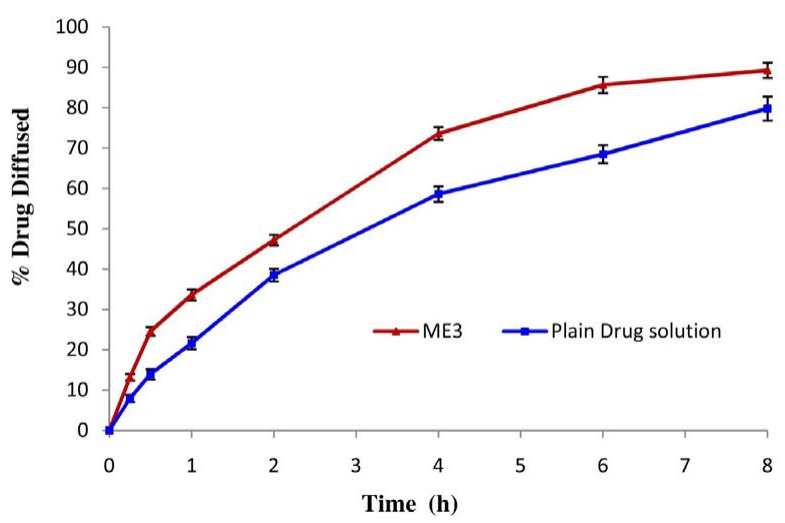

Figure 12: Permeation study of ME and plain drug solution through rat intestine.
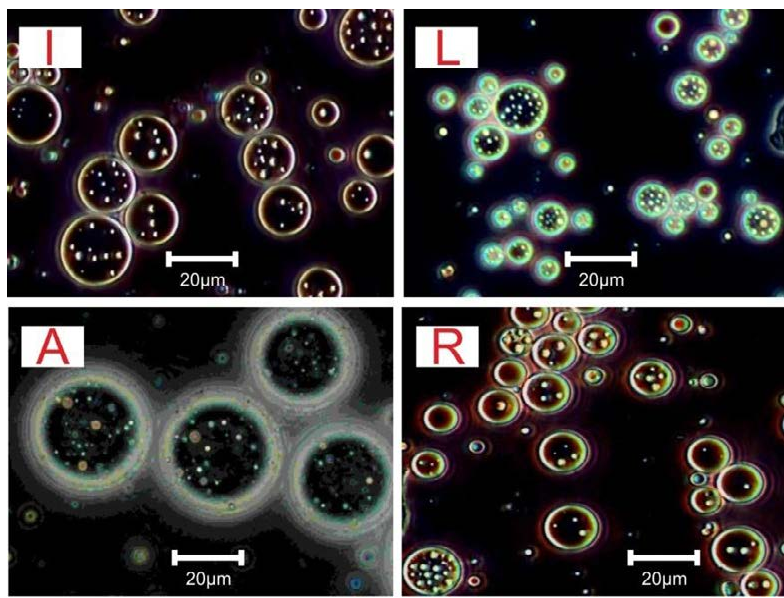

Figure 13: Optical photographs (40X) of MEs for Initial (I) and long term condition - $\left(25 \pm 2^{\circ} \mathrm{C} / 60 \pm 5 \% \mathrm{RH}\right)(\mathrm{L})$, accelerated condition - $\left(40 \pm 2^{\circ} \mathrm{C} / 75 \pm\right.$ $5 \% \mathrm{RH})(\mathrm{A})$, refrigerated condition- $\left(2-8^{\circ} \mathrm{C}\right)(\mathrm{R})$ after 3 months stability.

ratio (Er) was found to be 1.14, clearly indicating the enhancement in permeation by incorporating LD in ME, which is expected to enhance its absorption and bioavailability. LD is a BCS III drug, having high solubility but low permeability. Thus, formulation as $\mathrm{w} / \mathrm{o} / \mathrm{w}$ multiple emulsion could significantly enhance its permeability [4].

Stability studies: Influence of different storage conditions on the stability of MEs were assessed by analyzing them microscopically, visually and for PS, zeta potential, and EE. Significant changes in the PS and EE were observed during long term stability studies. Decrease in the globule size (from $18.89 \pm 2.46$ to $11.09 \pm 1.99 \mu \mathrm{m}$ ) and EE (from $79.18 \pm 2.79$ to $61.42 \pm 2.23$ ) was observed on storage at $25 \pm 2{ }^{\circ} \mathrm{C} / 60$ $\pm 5 \% \mathrm{RH}$. Decrease in the globule size might be due to the breaking of the ME into simple emulsion. This can also be attributed to continuous drug leakage from the MEs with time. This can also be ascribed to various phenomena such as coalescence, thinning or destruction of surfactant film or expulsion of internal aqueous phase, which are reported to be responsible for instability of MEs [10]. Drastic increase in particle size (from $18.89 \pm 2.46$ to $40.41 \pm 3.98$ ) and decrease in EE (from $79.18 \pm 2.79$ to $52.19 \pm 2.47$ ) was observed under accelerated conditions in 3 months. This increase was attributed to the coalescence of the globules. Loss of entrapped drug from ME lead to decrease in EE. This is expected as at elevated temperature, thermodynamic energy of drug molecules increases leading to a greater partitioning and diffusion of drug out of the hydrophilic layer of w/o/w emulsion causing loss of entrapped drug and reduction in EE [17]. These facts can be clearly seen from the microscopic view of the MEs as shown in Figure 13. There was major phase separation during accelerated condition, little in long term and no phase separation was seen at refrigerated conditions. Significant decrease in the viscosity of the MEs was also observed on storage. No significant change in zeta potential was observed in all three conditions.

Apparently, the formulation demonstrated acceptable physical (No Phase separation or coalesence) as well as chemical (No Drug leakage or degradation) stability [45] when stored under refrigerated conditions.

\section{Conclusions}

Stable corn oil based w/o/w MEs containing Lisinopril dihydrate were developed by two step emulsification technique using different surfactants (Span 80/Span 83/Tween 20). Important parameters were preliminary screened by Plackett-Burman design and further optimised by Box Behnken design. Phase Volume Ratio (W:O) in 
Citation: Sawant KK, Mundada VP, Patel VJ (2017) Development and Optimization of w/o/w Multiple Emulsion of Lisinopril Dihydrate Using Plackett Burman and Box-Behnken Designs. J Nanomed Nanotechnol 8: 422. doi: 10.4172/2157-7439.1000422

primary emulsion, Time for primary emulsification and Concentration of hydrophilic surfactant in secondary emulsion, were the three key variables affecting the preparation of stable ME formulations. Good correlation was obtained between actual and predicted values for the optimised formulation. The diffusion of drug across dialysis bag showed rapid release in beginning followed by a slower rate of release in 8 hours. Permeability study showed higher permeation rate from ME than from plain drug solution. Stability studies indicated that refrigeration condition was desirable for the storage of developed ME.

\section{Acknowledgments}

The Authors would like to acknowledge Torrent Research Centre, Ahmedabad, India for providing gift sample of Lisinopril Dihydrate.

\section{Conflict of Interest}

The authors report no conflict of interest.

\section{References}

1. Florence AT, Omotosho JA, Whateley TL (1989) Multiple w/o/w emulsions as drug vehicles, in Controlled release of drugs: polymers aggregate systems. VCH Publishers: New York, pp: 163-184.

2. Florence AT, Whitehill D (1982) The formulation stability of multiple emulsions. Int J Pharm 11: 277-308.

3. Omotosho JA, Florence AT, Whateley TL (1990) Absorption lymphatic uptake of 5 -fluorouracil in the rat following oral administration of w/o/w multiple emulsions. Int J Pharm 61: 51-56.

4. Koga K, Takarada N, Takada K (2010) Nano-sized water-in-oil-in-wate emulsion enhances intestinal absorption of calcein, a high solubility low permeability compound. Eur J Pharm Biopharm 74: 223-232.

5. Nakhare S, Vyas SP (1995) Prolonged Release Multiple Emulsion Based System Bearing Rifampicin: In Vitro Characterisation. Drug Dev Ind Pharm 21 869-878.

6. Shichiri M, Shimizu Y, Yoshida Y, Kawamori R, Fukuchi M, et al. (1974) Entera absorption of water-in-oil-in-water insulin emulsions in rabbits. Diabetologia 10: 317-321.

7. Khopade AJ, Jain NK (2008) Surface modified Fine Multiple Emulsions for AntiCancer Drug Delivery in Multiple Emulsion: Technology Applications. Wiley online.

8. Kim C, Kim S, Shin S, Kim KM, Oh X, et al. (1995) Preparation characterization of cytarabine-loaded w/o/w multiple emulsions. Int J Pharm 124: 61-68.

9. Onuki Y, Morishita M, Takayama K (2004) Formulation optimization of waterin-oil-water multiple emulsion for intestinal insulin delivery. J control Release 97: 91-99.

10. Paul S, Kumar A, Yedurkar P, Sawant K (2013) Design development of multiple emulsions for enhancement of oral bioavailability of acyclovir. Drug Dev Ind Pharm 39: 1809-1817.

11. http://www.accessdata.fda.gov/drugsatfda_docs/label/2014/019777s064lbl.pdf.

12. O'Neil MJ (2001) Lisinopril Dihydrate in The Merck Index - An Encyclopedia of Chemicals Drugs Biologicals. Whitehouse Station NJ.

13. Jackson EK (2006) Renin Angiotensin in Goodman Gilman's: The Pharmacological Basis of Therapeutics. McGraw-Hill: New York.

14. Kumar A, Sawant KK (2014) Application of multiple regression analysis in optimization of anastrozole-loaded PLGA nanoparticles. J Microencapsul 31 : 105-114

15. Negi LM, Jaggi M, Talegaonkar S (2013) A logical approach to optimize the nanostructured lipid carrier system of irinotecan: efficient hybrid design methodology. Nanotechnology 24: 1-14

16. Lewis GA, Mathieu D, Rodger PTL (1999) Pharmaceutical experimental design New York: Marcel Dekker Inc.

17. Aditya N, Ravi PR, Avula US, Vats R (2014) Poly (epsilon-caprolactone) nanocapsules for oral delivery of raloxifene: process optimization by hybrid design approach, in vitro in vivo evaluation. J Microencapsul 31: 508-518.

18. Gupta S, Chavhan S, Sawant KK (2011) Self-nanoemulsifying drug delivery system for adefovir dipivoxil: Design, characterization, in vitro ex vivo evaluation. Colloids Surfaces A 392: 145-155.
19. Aslan N, Cebeci Y (2007) Application of Box-Behnken design response surface methodology for modeling of some Turkish coals Fuel. My science work 86 90-97.

20. Derringer G, Suich R (1980) Simultaneous Optimization of Several Response Variables. J Quality Tech 12: 214-220.

21. http://www.drugbank.ca/drugs/DB00722

22. Yadav KS, Sawant KK (2010) Modified nanoprecipitation method for preparation of cytarabine-loaded PLGA nanoparticles. AAPS PharmSciTech 11: 1456-1465.

23. Tirnaksiz F, Kalsin O (2005) A topical w/o/w multiple emulsions prepared with Tetronic 908 as a hydrophilic surfactant: formulation, characterization release study. J Pharm Pharm Sci 8: 299-315.

24. Chavda H (2013) Self-nanoemulsifying Powder of Isotretinoin: Preparation Characterization. J Powder Technol.

25. Chakraborti CK, Sahoo S, Behera PK (2014) Role of different biodegradable polymers on the permeability of ciprofloxacin. J Adv Pharm Technol Res 5 : 140-146.

26. Patel MR (2009) Effect of formulation components on the in vitro permeation of microemulsion drug delivery system of fluconazole. AAPS PharmSciTech 10: $917-923$

27. Sawant KK, Rajesh KS (2010) Multiple Emulsion, in Vesicular \& Particulate Drug Delivery Systems. Murthy RSR, Editor Career Publications 141-166.

28. Pandey AP, Karande KP, Sonawane RO, Deshmukh PK (2014) Applying quality by design $(\mathrm{QbD})$ concept for fabrication of chitosan coated nanoliposomes. Liposome Res 24: 37-52

29. Kudarha R, Dhas NL, Pandey A, Belgamwar VS, Ige PP (2014) Box-Behnken study design for optimization of bicalutamide-loaded nanostructured lipid carrier: stability assessment. Pharm Dev Technol 20: 608-618.

30. Adinarayana K, Ellaiah P (2002) Response surface optimization of the critical medium components for the production of alkaline protease by a newly isolated Bacillus sp. J Pharm Pharm Sci 5: 272-278.

31. Bolton S, Bon C (2003) Pharmaceutical Statistics: Practical Clinical Applications. Taylor Francis.

32. Harrington JEC (1965) the Desirability Function. Industrial Quality Control 21 494-499.

33. Geiger S, Tokgoz S, Fructus A, Jager-Lezer N, Seiller M, et al. (1998) Kinetics of swelling-breakdown of a W/O/W multiple emulsions: possible mechanisms for the lipophilic surfactant effect. J Control Release 52: 99-107.

34. Redhead HM, Davis SS, Illum L (2001) Drug delivery in poly(lactide-coglycolide) nanoparticles surface modified with poloxamer 407 poloxamine 908 : in vitro characterisation in vivo evaluation. $\mathrm{J}$ control release $70: 353-363$.

35. Tang SY, Sivakumar M (2012) Design evaluation of aspirin-loaded water-in-oilin-water submicron multiple emulsions generated using two-stage ultrasonic cavitational emulsification technique. Asia Pac J Chem Eng 7: S145-S156.

36. Joshi SA, Chavhan SS, Sawant KK (2010) Rivastigmine-loaded PLGA PBCA nanoparticles: Preparation, optimization, characterization, in vitro pharmacodynamic studies. Eur J Pharm Biopharma 76: 189-199.

37. Feng SS, Huang G (2001) Effects of emulsifiers on the controlled release of

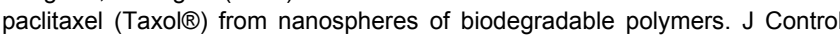
Release 71: 53-69.

38. Abdelbary G, Fahmy RH (2009) Diazepam-loaded solid lipid nanoparticles: design characterization. AAPS PharmSciTech. 10: 211-219.

39. Grossiord JL, Seiler M (1999) Multiple emulsions: structure properties applications. Editions de Sante.

40. Ozer O, Muguet V, Roy E, Grossiord JL, Seiller M (2000) Stability study of W/O/W viscosified multiple emulsions. Drug Dev Ind Pharm 26: 1185-1189.

41. Vasiljević DD, Parojčić JV, Primorac MM, Vuleta GM (2009) Rheological droplet size analysis of W/O/W multiple emulsions containing low concentrations of polymeric emulsifiers. J Serb Chem Soc 74: 801-816.

42. Pays K, Giermanska-Kahn J, Pouligny B, Bibette J, Leal-Calderon F, et al (2002) Double emulsions: how does release occur? J Control Release 79 193-205. 
Citation: Sawant KK, Mundada VP, Patel VJ (2017) Development and Optimization of w/o/w Multiple Emulsion of Lisinopril Dihydrate Using Plackett Burman and Box-Behnken Designs. J Nanomed Nanotechnol 8: 422. doi: 10.4172/2157-7439.1000422

Page 11 of 11

43. Raynal S (1993) A topical W/O/W multiple emulsion containing several active substances: formulation, characterization study of release. $\mathrm{J}$ control release 26: $129-140$

44. Magdassi S, Garti N (1986) A kinetic model for release of electrolytes from
W/O/W multiple emulsions. J control release 3: 273-277.

45. Khopade A, Jain N (1998) A stable multiple emulsion system bearing isoniazid: preparation characterization. Drug Dev Ind Pharm 24: 289-293. 